\title{
Antimicrobial Activity of Arugula (Eruca Sativa) Leaves on Some Pathogenic Bacteria
}

\author{
Samar Qaddoumi ${ }^{1} \&$ Nasser El-Banna ${ }^{2}$ \\ ${ }^{1}$ National Agricultural Research Center (NARC), Alba'qa, Jordan \\ ${ }^{2}$ Medical Laboratory Sciences, College of Sciences, Al-Balqa Applied University, Al-salt, Jordan \\ Correspondence: Samar Qaddoumi, National Center for Agricultural Research and Extension (NCARE), Alba'qa, \\ Jordan. E-mail: qaddoumi_99@yahoo.com
}

\author{
Received: March 7, $2019 \quad$ Accepted: March 26, $2019 \quad$ Online Published: May 4, 2019 \\ doi:10.5539/ijb.v11n3p10 URL: https://doi.org/10.5539/ijb.v11n3p10
}

\begin{abstract}
Arugula (Eruca sativa) is a green leafy vegetable; whose flowers, seed pods and seeds are edible. It's packed with vital nutrients that can help you step up your health without spending too much money. This study aims to fight pathogenic bacteria whether they affect plants or humans by stopping their growth and work as antibiotics. In the present study, water extract of Arugula leaves was effective against Escherichia coli HAS 11 (19mm) and Staphylococcus aureus HAS 1 (12mm), but no activity was observed against Erwinia amylovora HAS 12 and Bacillus cereus HAS 2. In the case of ethyl acetate extract, no antimicrobial activity against tested microorganisms, Staphylococcus aureus HAS 1, Bacillus cereus HAS 2, Escherichia coli HAS 11 and Erwinia amylovora HAS 12 was seen.
\end{abstract}

Keywords: Arugula, Antimicrobial Activity, Eruca Sativa

\section{Introduction}

Nature has been a source of medicinal agents for thousands of years and an impressive number of modern drugs have been isolated from natural sources; much of this isolation was based on the uses of these agents in traditional medicine (Cragg \& Newman, 2001). The study of biologically active compounds from natural sources has always been of great interest to scientists looking for new sources of useful drugs for treating infectious diseases. In the present study, the in vitro effect of Eruca sativa leaf extracts (which is commonly known as Rocket) on the survival and growth of certain gram positive and gram negative bacterial strains was investigated. It belongs to the Brassica plant family (Cruciferae), and is immensely used as vegetable and spice. It originated in Mediterranean region and now is found around the word (Al-qurainy et al., 2010).

The aim of the present study was to assess the in vitro antibacterial activity of medicinal plants extracts of $E$. sativa leaves.

\section{Materials and Methods}

\subsection{Bacterial Strain}

The antibacterial activity was screened against two Gram-positive bacteria (Staphylococcus aureus and Bacillus cereus) and two Gram-negative bacteria (Escherichia coli Erwinia spp.) were used as test microorganisms.

\subsection{Sample Collection and Preparation of Arugula Leaves Extract}

The arugula leaves were obtained from the market in Amman, Jordan. Samples were cleaned, dried in the drying oven at $50^{\circ} \mathrm{C}$ during $48 \mathrm{~h}$ and ground extraction. Ethyl acetate and water were used foe extraction of the active material. The extracts were filtered using Buckner funnel and Whatmann's No. 1 filter paper. Extracts were kept at $40^{\circ} \mathrm{C}$ to preserve the antibacterial property before they were used for agar diffusion assay.

\subsection{Determination of Antimicrobial Activity}

All the bacteria tested were grown on Mueller Hinton Agar. The antibacterial activity of the extracts (ethyl acetate and aqueous extracts) were measured by a diffusion test using Mueller-Hinton agar previously inoculated with $1 \mathrm{~mL}$ of $24 \mathrm{~h}$ old of bacterial suspension $\left(10^{6} \mathrm{CFU} / \mathrm{mL}\right)$ (Changwei et al., 2008). Wells in the agar media were prepared and filled with $20 \mu \mathrm{L}$ of extract. The plates were incubated at $4{ }^{\circ} \mathrm{C}$ for $2 \mathrm{~h}$ to allow diffusion of the active 
compounds in the medium (Tagg \& Mcgiven, 1971). Negative controls were prepared using the same solvent employed to dissolve the plant extract. Tetracycline was used as positive controls. Incubation of plates was performed at $37^{\circ} \mathrm{C}$ for $24 \mathrm{~h}$. Inhibition zones in $\mathrm{mm}$ around wells were measured. The antibacterial activity was expressed as the diameter of inhibition zones produced by the extract against test microorganisms. The experiment was repeated in triplicate and the mean of diameter of the inhibition zones was calculated.

\section{Results}

Plants have antimicrobial active compounds found in various plant parts such as stems, roots, leaves, bark, flowers or fruits and seeds. Thus, the medicinal and herbal plants have assumed greater importance in recent days, due to the tremendous potential that they offer in formulating new drugs against pathogenic bacteria (Malik, 2015). Eruca sativa was investigated in this study.

Figures 1 and 2, 3 and 4 present diameters of inhibition zones exerted by the extracts and the standard towards tested microorganisms. Water extract of Arugula leaves was effective against Escherichia coli HAS 11 (19mm) and Staphylococcus aureus HAS $1(12 \mathrm{~mm})$ as shown in Figures 1 and 2, respectively, but no activity was observed against Erwinia amylovora HAS 12 (Figure 3) and Bacillus cereus HAS 2 (Figure 4). In the case of Ethyl acetate extract, no antimicrobial activity against tested microorganisms, Staphylococcus aureus HAS 1 (Figure 5), Bacillus cereus HAS 2 (Figure 6), Escherichia coli HAS 11 (Figure 7) and Erwinia amylovora HAS 12 (Figure 8) was seen.

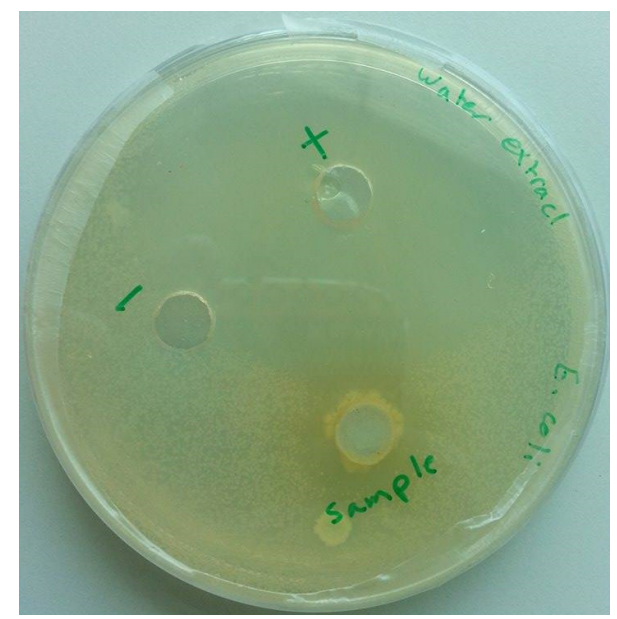

Figure 1. The antimicrobial activity of water extract of leaves of Eruca sativa against Escherichia coli HAS 11

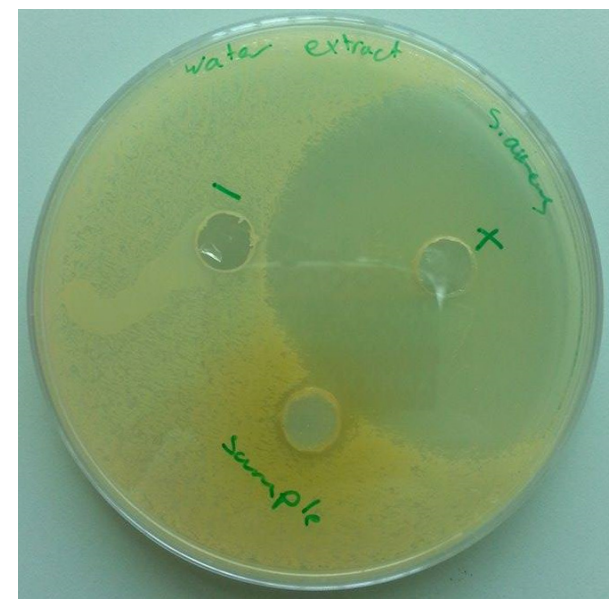

Figure 2. The antimicrobial activity of water extract of leaves of Eruca sativa against Staphylococcus aureus HAS 


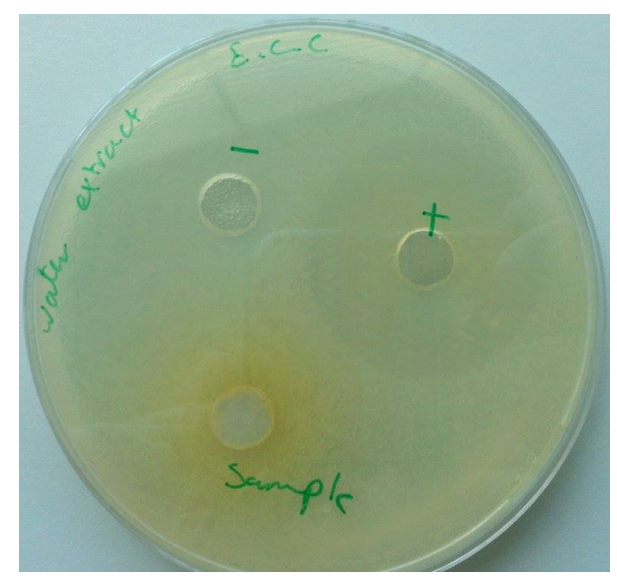

Figure 3. The antimicrobial activity of water extract of leaves of Eruca sativa against Erwinia amylovora HAS 12

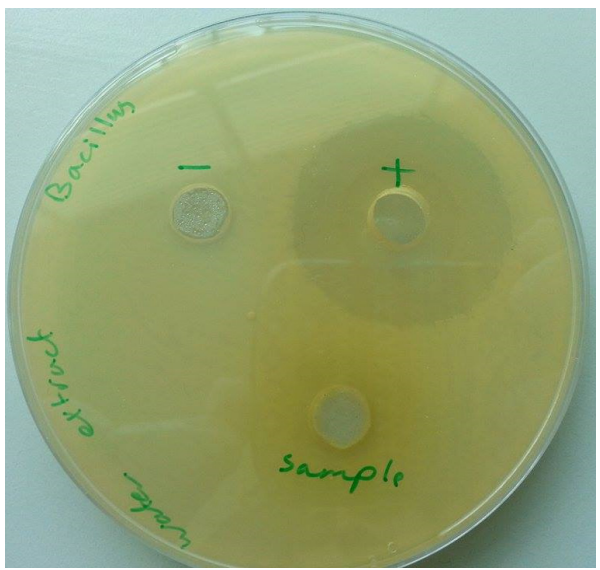

Figure 4. The antimicrobial activity of water extract of leaves of Eruca sativa against Bacillus cereus HAS 2

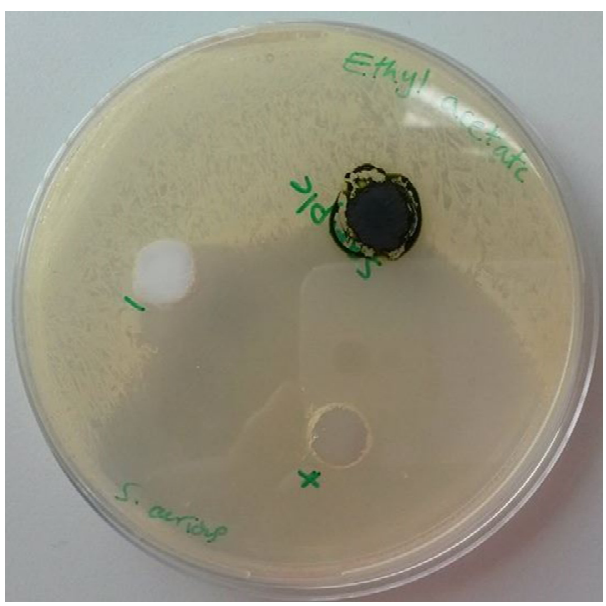

Figure 5. The antimicrobial activity of ethyl acetate extract of leaves of Eruca sativa against Staphylococcus aureus HAS 1 


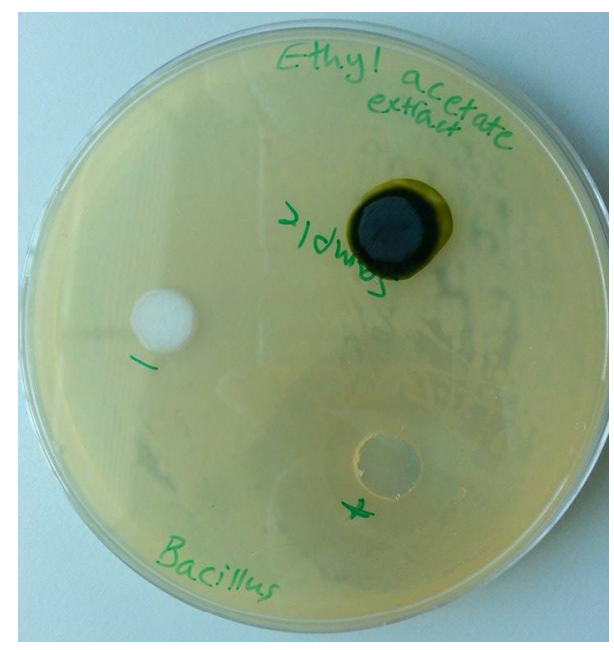

Figure 6. The antimicrobial activity of ethyl acetate extract of leaves of Eruca sativa against Bacillus cereus HAS 2

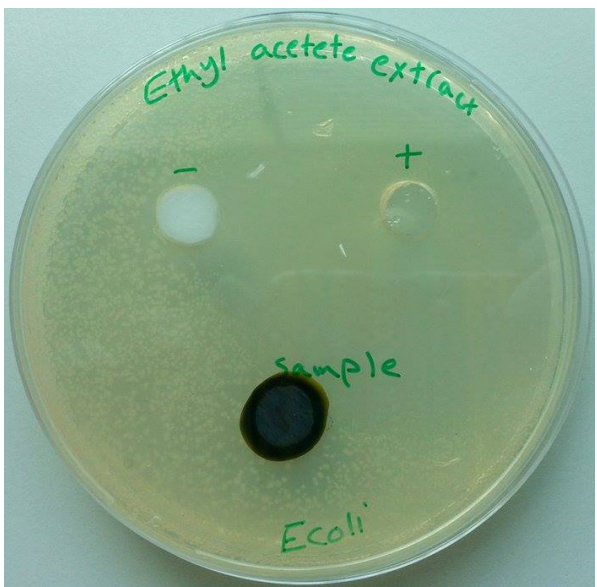

Figure 7. The antimicrobial activity of ethyl acetate extract of leaves of Eruca sativa against Escherichia coli HAS 11

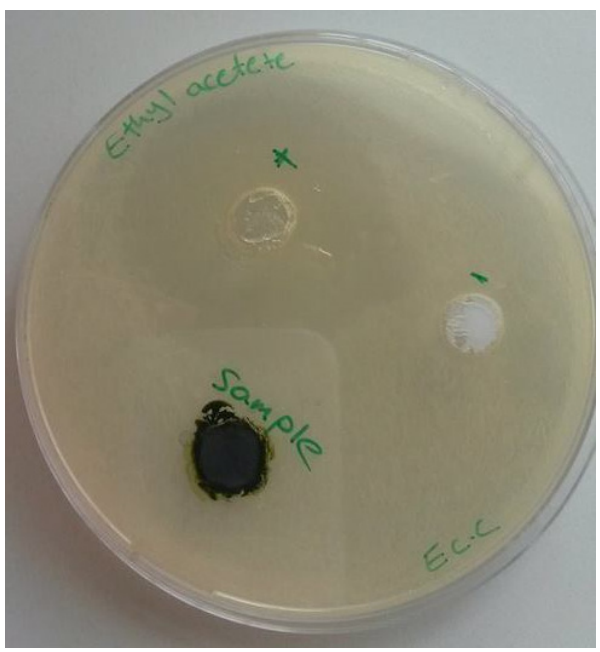

Figure 8. The antimicrobial activity of ethyl acetate extract of leaves of Eruca sativa against Erwinia amylovora HAS 12 


\section{Discussion}

In the present study on the antimicrobial activity of water and ethyl acetate extract of Arugula leaves against tested microorganisms, it was found inhibitory action of water extract against Escherichia coli HAS 11 (19mm) and Staphylococcus aureus HAS $1(12 \mathrm{~mm})$ as shown in Figures 1 and 2, respectively. While no activity against Erwinia amylovora HAS 12 (Figure 3) and Bacillus cereus HAS 2 (Figure 4) was observed. The ethyl acetate extract shows no antimicrobial activity against Staphylococcus aureus HAS 1 (Figure 5), Bacillus cereus HAS 2 (Figure 6), Escherichia coli HAS 11 (Figure 7) and Erwinia amylovora HAS 12 (Figure 8).

In latest years, Rocket plant (Eruca sativa) has gotten more value as avegetable, and spice around the world, further it is considered to be an important chemoprotective plant. The rocket can be eaten at various ontogenic phases (Okeke et al., 2005) The beneficial and positive usefulness of the phytochemicals existing in rocket on health have been notified by a numeral of scientific research studies. As well, the utilization of vegetables with green leaves has been related with the minimized hazard of cardiovascular diseases (CVD) (Joshipura et al., 2001). These advantageous effects have been linked to the variety of phytochemicals they consist of, such as vitamins $(\mathrm{C}, \mathrm{A})$, glucosinolates and flavonoids, all of which are found in large quantities in Brassicaceae crops (Jin et al., 2009; Bell, 2014). The rocket is believed to be an extremely good resource of antioxidants, as it includes phenolic compounds, glucosinolates carotenoids and degradation products like isothiocyanates (Villatoro-Pulido et al., 2012). Moreover, Eruca sativa has cytoprotective, anti-inflammatory, anti-ulcer and anti-secretory action (Alqasoumi, 2009; Khan, 2007; Heimler et al., 2007).

The sensitivity of Escherichia coli HAS 11 and Staphylococcus aureus HAS 1 to arugula leaves consistent with published data about, but the results are difficult to compare because literature assays were carried out arugula seeds (Malik, 2015). It found a remarkable antibacterial effect of extracts of Eruca sativa seeds against $S$. aureus (one of the most common of the Gram-positive bacteria causing food poisoning) and B. cereus.

It appears that overall the microorganisms were not as sensitive to the ethyl acetate extract compared with the water extract as determined by diffusion. The reasons for this could be that all of the identified components from Arugula active against microorganisms, water soluble compound, is most often obtained through initial water extraction.

Antimicrobial activity of Eruca sativa leaves was not previously reported. Most researches are about its seeds and flowers (Malik, 2015; Koubaa et al., 2015)

Eruca sativa leaves may be useful in cases where prolonged use of antibiotics encourage development of opportunistic infections.

\section{Conclusion}

In conclusion, the data obtained in this study demonstrate that the use of arugula leaves as nutraceuticals may lower the risk of microbial infections, particularly in the intestinal and respiratory tract, mainly due to the protective action provided by its extract. Hence, this extract can be recommended for therapeutic purposes and be used as an alternative medicine.

\section{Conflict of interests}

The authors declare that there is no conflict of interests regarding the publication of this paper.

\section{References}

Alqasoumi, S., Al-Sohaibani, M., Al-Howiriny, T., Al-Yahya, M., \& Rafatullah, S. (2009). Rocket "Eruca sativa". A salad herb with potential gastric anti-ulcer activity. World Journal of Gastroenterology, 15, 1958-1965.

Al-qurainy, F., Alameri, A., \& Khan, S. (2010). RAPD profile for the assessment of genotoxicity on a medicinal plant; Eruca sativa. J. Med. Plants. Res., 4(7), 579-586.

Bell, L., \& Wagstaff, C. (2014). Glucosinolates, myrosinase hydrolysis products, and flavonols found in rocket (Eruca sativa and Diplotaxis tenuifolia). Journal of Agriculture Food Chemistry, 62, 4481-4492.

Changwei, A., Anpin, L., Abdelnaser, A., Tran, D., \& Shinkichi, T. (2008). Evaluation of antioxidant and antibacterial activities of Ficus microcarpa L. fil. extract. Food Control, 19, 940-948.

Cragg, G., \& Newman, D. (2001). Medicinals for the millennia. Ann NY Acad Sci., 953, 3-25.

Heimler, D., Isolani, L., Vignolini, P., Tombelli, S., \& Romani, A. (2007). Polyphenol content and antioxidative activity in some species of freshly consumed salads. Journal of Agriculture Food Chemistry, 55, 1724-1729. 
Jin, J., Koroleva, O., Gibson, T., Swanston, J., Magan, J., \& Zhang, Y. (2009). Analysis of phytochemical composition and chemoprotective capacity of rocket (Eruca sativa and Diplotaxis tenuifolia) leafy salad following cultivation in different environments. Journal of Agriculture Food Chemistry, 57, 5227-5234.

Joshipura, K., Hu, F., Manson, J., Stampfer, M., Rimm, E., \& Speizer, F. (2001). The effect of fruit and vegetable intake on risk for coronary heart disease. Annual Internal Medicin., 134, 1106-1114.

Khan, H., \& Khan, M. (2014). Antiulcer Effect of Extract/Fractions of Eruca sativa: Attenuation of Urease Activity. Journal of Evidaned Based Complement Alternative Medicine, 19, 176-180.

Koubaa, M., Driss, D., Bouaziz, F., Ghorbel, R., \& Chaabouni, S. (2015). Antioxidant and antimicrobial activities of solvent extract obtained from rocket (Eruca sativa L.) flowers. Free Rad Antiox, 5, 29-34.

Malik, N. (2015). Antibacterial Activity of Olive (Olea europaea) Leaves and Arugula (Eruca sativa) Seeds Extract. International Journal of Pharmacognosy and Phytochemical Research, 7(2), 307-310.

Okeke, I., Laxminarayan, R., \& Bhutta, Z. (2005). Antimicrobial resistance in developing countries. Part 1: Recent trends and current status. Lancet Infect Disease, 5, 481-493.

Tagg, J., \& Mcgiven, A. (1971). Assay system for bacteriocins. Applied Microbiology, 21, 943-944.

Villatoro-Pulido, M., Font, R., Saha, S., Obregon-Cano, S., Anter, J., \& Munoz-Serrano, A. (1012). In vivo biological activity of rocket extracts (Eruca vesicaria subsp. sativa and sulforaphane. Food and Chemical Toxicology Nutrients, 50, 1384-1392.

\section{Copyrights}

Copyright for this article is retained by the author(s), with first publication rights granted to the journal.

This is an open-access article distributed under the terms and conditions of the Creative Commons Attribution license (http://creativecommons.org/licenses/by/4.0/). 